\title{
Aspects of Weyl supergravity
}

\section{Sergio Ferrara, ${ }^{a, b, c}$ Alex Kehagias ${ }^{d}$ and Dieter Lüst ${ }^{a, e, f}$}

${ }^{a}$ Theory Department, CERN,

1211 Geneva 23, Switzerland

${ }^{b}$ INFN, Laboratori Nazionali di Frascati, Via Enrico Fermi 40, 00044 Frascati, Italy

${ }^{c}$ Department of Physics and Astronomy and Mani L. Bhaumik Institute for Theoretical Physics, University of California, Los Angeles, Los Angeles CA 90095-1547, U.S.A.

${ }^{d}$ Physics Division, National Technical University of Athens, 15780 Zografou Campus, Athens, Greece

${ }^{e}$ Arnold-Sommerfeld-Center for Theoretical Physics, Ludwig-Maximilians-Universität, 80333 München, Germany

${ }^{f}$ Max-Planck-Institut für Physik, Werner-Heisenberg-Institut, 80805 München, Germany

E-mail: Sergio.Ferrara@cern.ch, kehagias@central.ntua.gr, luest@mppmu .mpg.de

ABSTRACT: In this paper we study the spectrum of all conformal, $\mathcal{N}$-extended supergravities $(\mathcal{N}=1,2,3,4)$ in four space-time dimensions. When these theories are obtained as massless limit of Einstein plus Weyl ${ }^{2}$ supergravity, the appropriate counting of the enhanced gauge symmetries allow us to derive the massless spectrum which consist of a dipole ghost graviton multiplet, a $\mathcal{N}$-fold tripole ghost gravitino, the third state belonging to a spin $3 / 2$ multiplet and a residual vector multiplet present for non-maximal $\mathcal{N}<4$ theories. These theories are not expected to have a standard gravity holographic dual in five dimensions.

KEYwORDS: Conformal and W Symmetry, Extended Supersymmetry, Supergravity Models

ARXIV EPRINT: 1806.10016 


\section{Contents}

1 Introduction 1

2 Bosonic Weyl gravity 2

2.1 Massive theory 2

2.2 Massless theory 4

2.3 Higher tensors 4

$\begin{array}{lll}3 \mathcal{N}=1 & \text { super-Weyl theory } & 7\end{array}$

3.1 Massive theory 7

3.2 Massless theory 8

$\begin{array}{ll}3.3 \text { Higher tensors } & 10\end{array}$

$4 \mathcal{N}=2$ super-Weyl theory 12

$\begin{array}{lll}4.1 & \text { Massive theory } & 12\end{array}$

4.2 Massless theory 13

$5 \mathcal{N}=3$ super-Weyl theory 14

5.1 Massive theory 14

$\begin{array}{lll}5.2 & \text { Massless theory } & 15\end{array}$

$6 \mathcal{N}=4$ super-Weyl theory $\quad 16$

$\begin{array}{ll}6.1 \text { Massive theory } & 16\end{array}$

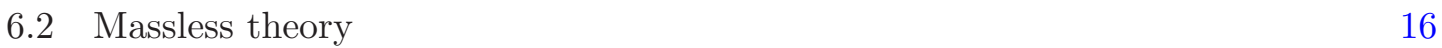

$\begin{array}{llc}7 & \text { Conclusions and outlook } & 18\end{array}$

\section{Introduction}

There is recently a renewed interest in higher curvature theories. These are theories of the general form $R+\mathcal{R}^{n}$, where $R$ is the standard Einstein term and $\mathcal{R}^{n}$ denotes collectively $n^{\text {th }}$ power of the Riemann, Ricci, Weyl tensors or the curvature scalar [1-10]. A recent discussion on this class of models can be found in [11]. In particular, the $R+R^{2}$ supergravity has been studied $[12,13]$, especially in connection to the inflationary dynamics [14-27]. In fact, the $R+R^{2}$ theory, known as the Starobinsky model [28, 29] for inflation, propagates besides the usual massless graviton, an additional massive spin- 0 state, known as the "scalaron field" or the so called "no-scale field". It is this mode that can be identified with the inflaton field and makes the theory so appealing as inflation is driven entirely by gravity itself and not by some external scalar field. Furthermore, the $R+R^{2}$ theory can also be embedded consistently in supergravity, whereas the linearized $\mathcal{N}=1$ theory has been analysed in [30] and the $\mathcal{N}=2$ in [31]. 
On the other hand the $R+R^{2}$ theory does not include all possible quadratic curvature theories. Indeed, a second independent quadratic curvature invariant is the square of the Weyl tensor, whereas terms quadratic in the Riemann (or Ricci) tensor can be traded for a Weyl square term and the 4D Gauss-Bonnet topological term. However, when the Weyl square term is included in the low-energy gravitational effective action, the spectrum changes and includes an additional massive spin-2 ghost $[1,2] .^{1}$

In the present paper we first discuss the massive Weyl ${ }^{2}$ theory and its supersymmetric extensions, namely $\mathcal{N}$-extended $\mathrm{Weyl}^{2}$ supergravities, which contain not only the $\mathrm{Weyl}^{2}$ term but also the Einstein term $m^{2} R .^{2}$ The latter can therefore be seen as a mass deformation of the massless theory. In addition, due to the relation between massive and massless Weyl supergravity, the bound $\mathcal{N}=8$ of Poincare supergravity tranfers to $\mathcal{N}=4$ in the case of Weyl supergravity [32].

Second, we are particularly interested in the massless limit $m \rightarrow 0$ of the $m^{2} R+\mathrm{Weyl}^{2}$ theory in conformal supergravity [33-36]. Although these theories contain propagating ghosts, they are nevertheless very interesting, since in the massless limits, i.e. in the absence of the Einstein term, they provide unique examples of (super)conformal gravitational theories with up to four derivative terms. Namely in the limit $m \rightarrow 0$ the spectrum gets re-organized and the symmetry gets enhanced, namely from (super) Poincare is enhanced to (super) conformal. In additional also the R-symmetries become local gauge symmetries. Furthermore in this limit, there are various primary operators, like the Weyl tensor itself. Another conformal tensor is the Bel-Robinson tensor, which is basically the square of the Weyl tensor. They have a well-defined conformal weight, i.e. transform under conformal transformations in a homogeneous way. This is true in the massive case and also in the limit of zero mass, i.e. the limit is continuous with respect to the scaling weights. Furthermore the Bel-Robinson tensor is conserved in the massive theory on Ricci-flat spaces.

The paper is organized as follows: in section 2 we describe the bosonic Weyl ${ }^{2}$ gravity, its spectrum and its higher dimensional operators. In section 3 we discuss the spetcrum of the super-Weyl theory. Here we again provide some details of the higher dimensional operators using $\mathcal{N}=1$ superfield language. In sections 4,5 and 6 , the spectra of the $\mathcal{N}=2, \mathcal{N}=3$ and $\mathcal{N}=4$ super-Weyl $^{2}$ theories, respectively, are determined. We close in section 7 with some discussions and expectations on the holographic duals of the (super)conformal Weyl ${ }^{2}$ gravity.

\section{Bosonic Weyl gravity}

\subsection{Massive theory}

Let us first recall the bosonic Einstein plus (Weyl) ${ }^{2}$ gravity theory in four dimensions. More details can be e.g. found in $[11,40]$. The action up to four orders in derivatives has

\footnotetext{
${ }^{1}$ It should be noted that the problem with such ghosts states is that one cannot maintain at the same time unitarity and forward propagation in time of positive energy states. Indeed with the opposite $+i \epsilon$ choice one propagates negative energies forward in time but unitarity and the optical theorem is preserved,

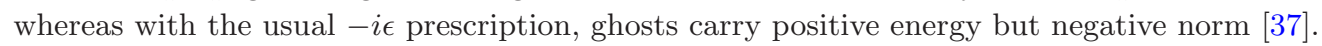

${ }^{2} \mathrm{~A}$ very interesting double copy construction of $\mathrm{Weyl}^{2}$ (super) gravities was recently provided in [38, 39].
} 
the following form:

$$
S=\int d^{4} x \sqrt{-g}\left(\frac{a}{2} W_{\mu \nu \rho \sigma} W^{\mu \nu \rho \sigma}+\kappa^{2} R\right) .
$$

Here $W_{\mu \nu \rho \sigma}=R_{\mu \nu \rho \sigma}-g_{\mu[\sigma} R_{\rho] \nu}-g_{\mu[\rho} R_{\sigma] \nu}-R / 3 g_{\mu[\rho} g_{\sigma] \nu}$ is the Weyl tensor. The Weyl ${ }^{2}-$ term in the action possesses conformal invariance as it is invariant under the conformal transformation

$$
g_{\mu \nu} \rightarrow \widehat{g}_{\mu \nu}=\Omega^{2} g_{\mu \nu},
$$

which leaves the Weyl tensor inert

$$
\widehat{W}_{\nu \rho \sigma}^{\mu}=W_{\nu \rho \sigma}^{\mu} .
$$

However the Einstein-term is not invariant under conformal transformations, since $R$ transforms under conformal trasformations as:

$$
\widehat{R}=\Omega^{-2} R-6 \Omega^{-3} g^{\mu \nu} \nabla_{\mu} \nabla_{\nu} \Omega .
$$

Therefore the Einstein-term can be regarded as the mass term in this theory, i.e. a mass deformation, which explicitly breaks conformal invariance.

The equations of motion which follow from (2.1) are written as

$$
B_{\mu \nu}+\frac{2 \kappa^{2}}{a} G_{\mu \nu}=0
$$

where $B_{\mu \nu}$ is the Bach tensor

$$
B_{\mu \nu}=\nabla^{\rho} \nabla_{\sigma} W_{\mu \rho \nu}^{\sigma}+\frac{1}{2} R^{\rho \sigma} W_{\rho \mu \sigma \nu},
$$

with

$$
\widehat{B}_{\mu \nu}=\Omega^{-2} B_{\mu \nu} .
$$

Note that the second term in $B_{\mu \nu}$ is needed such that the Bach tensor transforms with a uniform weight under conformal transformations. The Bach tensor is symmetric, traceless due to conformal invariance and divergence-free (due to diff. invariance)

$$
B_{\mu}^{\mu}=0, \quad \nabla^{\mu} B_{\mu \nu}=0,
$$

and $G_{\mu \nu}$ is the Einstein tensor.

Now we can recall the propagating modes corresponding to this action. For this, one analyzes the poles in the propagators generated by its quadratic part. Specifically, there are two kinds of propagating modes $[1,2]$ :

(i) A massless helicity- \pm 2 graviton $g_{\mu \nu}$. This mode is independent of the couplings $a$ and $\kappa^{2}$ and it is the standard massless spin-two graviton.

(ii) A massive spin-two particle $w_{\mu \nu}$ with mass $\kappa^{2} / a$. It is related to the Weyl $l^{2}$ term in the action. In fact, this massive spin two particle is a ghost for $a>0$, destroying unitarity, or a tachyon for $a<0$, leading to an instability. We will call this part of the spectrum the non-standard sector of the theory.

Hence in summary, the Einstein plus (Weyl) ${ }^{2}$ gravity theory contains seven propagating degrees of freedom. 


\subsection{Massless theory}

In the following we consider the massless limit $\kappa=0$, which is a pure Weyl ${ }^{2}$ theory with action

$$
S_{\mathrm{Wey} l^{2}}=\frac{1}{2 g^{2}} \int d^{4} x \sqrt{-g} W_{\mu \nu \rho \sigma} W^{\mu \nu \rho \sigma}
$$

where $g^{2}=1 / a$ is a dimensionless coupling. Now the equations of motion are simply

$$
B_{\mu \nu}=0 .
$$

At the linearized level of the theory, the conformally invariant Weyl equation of motion simply looks like [41]

$$
\partial^{\mu} \partial^{\rho} W_{\mu \nu \rho \sigma}=0 \text {. }
$$

The pure Weyl ${ }^{2}$ possesses conformal invariance and it propagates six degrees of freedom [42]:

(i) The standard massless spin-two graviton, corresponding to a planar wave in Einstein gravity.

(ii) In the non-standard sector there is massless spin-two ghost particle, which corresponds to a non-planar wave. In addition there is a massless vector, which originates from the \pm 1 helicities of the massive $w_{\mu \nu}$ particle. However note that the helicity zero component of $w_{\mu \nu}$ does not correspond to a physical, propagating mode in the massless limit, since it can be gauged away by the conformal transformations (2.2).

\subsection{Higher tensors}

In this section we briefly discuss some higher tensors, which are also of interest in the massless Weyl ${ }^{2}$ theory. In fact, the aim of this discussion is to construct conformal operators, which can be coupled to spin-four fields (see also the concluding section of this paper). It is known in the literature that there are no totally symmetric, quadratic in the curvature and divergence-free four-index tensors of dimension four [43] in a generic background. However, there are dimension four, divergence-free tensors which are totally symmetric just in three-indices. It is also known that there exist a unique totally symmetric, traceless and divergence-free four-index tensor, on Ricci-flat spaces, which is the Bel-Robinson tensor [44-47]

$$
\begin{aligned}
T_{\mu \nu \rho \sigma} & =\frac{1}{4}\left(W_{\nu \mu}^{\lambda}{ }^{\kappa} W_{\lambda \sigma \rho \kappa}+\frac{1}{2} \epsilon_{\nu \tau \xi}^{\lambda} \epsilon_{\lambda \sigma}{ }^{\chi \psi} W_{\mu}^{\tau \xi}{ }_{\mu}^{\kappa} W_{\chi \psi \rho \kappa}\right) \\
& =\frac{1}{4}\left(W_{\nu \mu}^{\lambda}{ }^{\kappa} W_{\lambda \sigma \rho \kappa}+W_{\sigma \mu}^{\lambda}{ }^{\kappa} W_{\lambda \nu \rho \kappa}-\frac{1}{2} g_{\nu \sigma} W_{\mu}^{\lambda \tau}{ }^{\kappa} W_{\lambda \tau \sigma \kappa}\right) .
\end{aligned}
$$

This tensor is traceless $T_{\mu \nu \rho}^{\mu}=0$ and satisfies

$$
\nabla_{\mu} T_{\nu \rho \sigma}^{\mu}=\frac{1}{2}\left(R_{\nu \kappa \rho}^{\lambda} \nabla_{[\lambda} R_{\sigma]}^{\kappa}+R_{\nu \kappa \sigma}^{\lambda} \nabla_{[\lambda} R_{\rho]}^{\kappa}\right) .
$$


Hence in Einstein gravity, for Ricci-flat spaces, the Bel-Robinson tensor is divergence-free, $\nabla_{\mu} T_{\nu \rho \sigma}^{\mu}=0$. Of course, Ricci-flat manifolds are also Bach-flat but the contary is not true. For example, manifolds conformal to Einstein spaces have vanishing Bach tensor but nonvanishing Ricci. In addition, the Bel-Robinson tensor transforms under Weyl rescalings as

$$
T_{\rho \sigma}^{\mu \nu} \rightarrow \widehat{T}_{\rho \sigma}^{\mu \nu}=\Omega^{-4} T_{\rho \sigma}^{\mu \nu},
$$

and therefore it has conformal dimension $\Delta_{T}=4 .^{3}$ Let us recall here that in general, primary operators of spin $s$ and dimension $\Delta$ in $d$-dimensional unitary $C F T_{d}$, satisfy the unitarity bound $[48,49]$

$$
\Delta \geq d-2+s
$$

In terms of the twist $\tau$ defined by

$$
\tau=\Delta-s
$$

the unitarity bound (2.15) is written as

$$
\tau \geq d-2
$$

Primary operators that saturate the bound satisfy the equation $\partial_{\mu_{1}} J_{\mu_{2} \cdots \mu_{s}}^{\mu_{1}}=0$ and therefore correspond to conserved operators. In particular, for $d=4$ we find that $\tau=2$ for a conserved primary which is the case for the spin-two energy momentum tensor. ${ }^{4}$ However in our case the Bel-Robsinon operator has dimension $\Delta_{T}=4$ and $\operatorname{spin} s=4$, and therefore it has twist $\tau_{T}=0$. It follows that the Bel-Robinson operator violates the unitarity bound, which is expected, since we know that the $\mathrm{Weyl}^{2}$ contains ghosts and is therefore nonunitary. Nevertheless as the ghost states can be projected out by appropriate boundary conditions [9], we may still look for a tensor $J_{\mu \nu \rho \sigma}$ like the Bel-Robinson tensor that has the same symmetries with it, it is divergence-free for Bach-flat spaces and has twist $\tau=2$. If such a tensor exist, it could couple to a spin-4 field. In addition, the conformal dimension of $J_{\mu \nu \rho \sigma}$ should be

$$
\Delta_{J}=6
$$

in order to describe a spin-4 conserved operator in the CFT. It is natural to expect that, similarly to the energy momentum tenor, $J_{\mu \nu \rho \sigma}$ is quadratic in a tensor build out of the curvature and/or its derivatives of dimension $\Delta=3$. Such a tensor with $\Delta=3$ exists in conformal gravity and it is the Cotton tensor defined as

$$
C_{\mu \nu \rho}=\nabla_{\mu} S_{\nu \rho}-\nabla_{\nu} S_{\mu \rho}
$$

\footnotetext{
${ }^{3}$ The conformal dimension of a field in CFT is the weight under the Weyl transformation of this field with half its indices up and half down.

${ }^{4}$ In case the energy momentum tensor is non-conserved and has dimension $\Delta>2+s$, the corresponding bulk spin-two field becomes massive [50].
} 
where

$$
S_{\mu \nu}=R_{\mu \nu}-\frac{1}{6} g_{\mu \nu} R
$$

is the Schouten tensor. In particular, by using the second Bianchi identity, the Cotton tensor can be written as the divergence of the Weyl tensor

$$
C_{\mu \nu \rho}=\nabla_{\kappa} W_{\rho \mu \nu}^{\kappa}
$$

and therefore

$$
C_{[\mu \nu \rho]}=0 .
$$

We can now define a conserved 4-tensor quadratic in the Cotton tensor as follows. From eq. (2.19) we get the equation ${ }^{5}$

$$
\nabla_{[\lambda} C_{\rho \sigma] \kappa}=B_{\lambda \rho \sigma \kappa}
$$

with

$$
B_{\lambda \rho \sigma \kappa}=R_{\kappa[\rho \lambda}^{\nu} S_{\sigma] \nu}=R_{\kappa[\rho \lambda}^{\nu} R_{\sigma] \nu}=W_{\kappa[\rho \lambda}^{\nu} R_{\sigma] \nu}
$$

whereas, using eqs. (2.6), (2.21) we find

$$
\nabla^{\kappa} C_{\kappa \mu \nu}=A_{\mu \nu}
$$

with

$$
A_{\mu \nu}=B_{\mu \nu}-\frac{1}{2} R^{\kappa \lambda} W_{\mu \kappa \nu \lambda} .
$$

Multiplying eq. (2.23) by $C^{\lambda \rho \mu}$ and after some algebra we arrive at

$$
\nabla_{\lambda} J_{\alpha \mu \nu}^{\lambda}=H_{\alpha \mu \nu}
$$

where

$$
J_{\alpha \mu \nu}^{\lambda}=\frac{1}{4}\left(C_{\nu}^{\lambda \gamma} C_{\alpha \gamma \mu}+C_{\mu}^{\lambda \gamma} C_{\alpha \gamma \nu}-\frac{1}{2} \delta_{\alpha}^{\lambda} C_{\nu}^{\rho \sigma} C_{\rho \sigma \mu}\right) .
$$

and

$$
H_{\alpha \mu \nu}=\frac{1}{8}\left(C_{\nu}^{\gamma \lambda} B_{\alpha \lambda \gamma \mu}+C_{\mu}^{\gamma \lambda} B_{\alpha \lambda \gamma \nu}+2 A_{\mu}^{\gamma} C_{\alpha \gamma \nu}+2 A_{\nu}^{\gamma} C_{\alpha \gamma \mu}\right) .
$$

Note that under a conformal transformation with $\delta g_{\mu \nu}=2 \omega g_{\mu \nu}$, the Cotton tensor $C_{\mu \nu \rho}$ and the tensor $J_{\lambda \alpha \mu \nu}$ transform inhomogeneously under conformal transformations. In particular the transformation of the Cotton tensor turns out to be

$$
\delta C_{\mu \nu \rho}=2 \partial_{\sigma} \omega W_{\rho \mu \nu}^{\sigma},
$$

\footnotetext{
${ }^{5}$ To obtain eq. (2.23), the relation $\nabla_{\mu} \nabla_{\nu} V^{\rho}-\nabla_{\nu} \nabla_{\mu} V^{\rho}=R^{\rho}{ }_{\kappa \mu \nu} V^{\kappa}$ has been used.
} 
whereas, $J_{\lambda \alpha \mu \nu}$ transforms as

$$
\delta J_{\lambda \alpha \mu \nu}=-2 \omega J_{\lambda \alpha \mu \nu}+\frac{1}{2} \partial_{\tau} \omega\left(W_{\lambda \nu}^{\tau \gamma} C_{\gamma \alpha \mu}+W_{\gamma \alpha \nu}^{\tau} C_{\lambda \mu}^{\gamma}-\frac{1}{2} W_{\nu}^{\tau \rho \sigma} C_{\rho \sigma \mu}+\mu \leftrightarrow \nu\right) .
$$

This means that both $C_{\mu \nu \rho}$ and $J_{\lambda \alpha \mu \nu}$, although not Weyl invariant, are Weyl covariant [51] as their transformation under Weyl rescalings does not involve higher than first derivatives of the conformal factor. Therefore in order to obtain a conformal tensor one should use a Weyl-covariant derivative for the construction of an "improved" Cotton tensor, along the lines of [51].

It is easy to prove that in the linearized theory, the $J^{\lambda \mu \nu \rho}$ is divergence-free and has the correct conformal dimension $\Delta_{J}=6$

$$
\begin{aligned}
\partial_{\mu} J_{\rho \sigma}^{\mu \nu} & =0, \\
\widehat{J}_{\rho \sigma}^{\mu \nu} & =\Omega^{-6} J_{\rho \sigma}^{\mu \nu} .
\end{aligned}
$$

If (2.32) can be extended at the full non-linear level is not known to us.

\section{$3 \mathcal{N}=1$ super-Weyl theory}

\subsection{Massive theory}

Now we want to present the superfield versions of the bosonic Weyl ${ }^{2}$-action, first using $\mathcal{N}=1$ supersymmetry language. In conformal supergravity, one first introduces the superWeyl tensor $\mathcal{W}_{\alpha \beta \gamma}$, which is a chiral superfield, where $\alpha, \beta, \gamma=1,2$ are standard $\operatorname{SL}(2, \mathbf{C})$ spinor indices. $\mathcal{W}_{\alpha \beta \gamma}$ has spin $\left(\frac{3}{2}, 0\right)$, i.e. its highest component is a fermionic spin- $3 / 2$ field, but $\mathcal{W}_{\alpha \beta \gamma}$ also contains the spin-two field $g_{\mu \nu}$ with five degrees of freedom. The Weyl superfield $\mathcal{W}_{\alpha \beta \gamma}$ satisfies

$$
\mathcal{W}_{\alpha \beta \gamma}=\mathcal{W}_{(\alpha \beta \gamma)}, \quad\left(\mathcal{W}_{\alpha \beta \gamma}\right)^{*}=\overline{\mathcal{W}}_{\dot{\alpha} \dot{\beta} \dot{\gamma}}, \quad \overline{\mathcal{D}}_{\dot{\delta}} \mathcal{W}_{\alpha \beta \gamma}=0
$$

and it has the following pure bosonic contributions

$$
\mathcal{D}_{\gamma} \mathcal{W}_{\delta \epsilon \alpha} \mid=\frac{1}{6}\left(\frac{i}{4} \epsilon_{\gamma \delta} \mathcal{D}_{\epsilon \dot{\epsilon}} A_{\alpha}^{\dot{\epsilon}}-\frac{1}{4} \epsilon^{\beta} \epsilon_{\epsilon}{ }_{\gamma} W_{\delta \beta \alpha \rho}\right)+(\delta \epsilon \alpha),
$$

where $W_{\delta \beta \alpha \rho}$ and $A_{\alpha}^{\dot{\epsilon}}$ are the spinorial equivalent or the Weyl conformal tensor and the vector auxiliary of the $\mathcal{N}=1$ supergravity, and $(\delta \epsilon \alpha)$ denotes five terms obtained by symmetrization with respect to the fermionic indices $\delta, \epsilon$ and $\alpha$ [52].

The supersymmetric action of massive Weyl gravity is then written as

$$
\mathcal{L}_{\Phi, \mathcal{W}}=\int d^{2} \Theta 2 \mathcal{E} \mathcal{R}+4 \int d^{2} \Theta 2 \mathcal{E} \tau \mathcal{W}^{2}+\text { c.c. },
$$

where $\tau$ is the complex coupling

$$
\tau=\frac{1}{g^{2}}+i \alpha
$$


We then find that the bosonic sector of the action is

$$
\begin{aligned}
e^{-1} \mathcal{L}_{\mathcal{W}}= & \frac{1}{2} R+\frac{1}{3} A_{\mu} A^{\mu}-\frac{1}{3} u \bar{u}+\frac{\alpha}{2}\left(\frac{1}{2} R_{H P}^{2}-\frac{2}{3} F_{\mu \nu} F_{\rho \sigma} \epsilon^{\mu \nu \rho \sigma}\right) \\
& +\frac{1}{2 g^{2}}\left(W^{\mu \nu \rho \sigma} W_{\mu \nu \rho \sigma}-\frac{4}{3} F^{\mu \nu} F_{\mu \nu}\right)
\end{aligned}
$$

where $F_{\mu \nu}=\partial_{\mu} A_{\nu}-\partial_{\nu} A_{\mu}$ is the field strength of the supergravity vector $A_{\mu}, u$ is the auxiliary scalar and $R_{H P}^{2}=W_{\mu \nu \kappa \lambda} \epsilon^{\kappa \lambda \rho \sigma} W_{\rho \sigma}{ }^{\mu \nu}$ is the topological Hirzebruch-Pontryagin term. Note that in Weyl supergravity, the vector $A_{\mu}$ is dynamical as it has a kinetic term of the form $F^{\mu \nu} F_{\mu \nu}$.

Now we are ready to determine the spectrum of the massive $\mathcal{N}=1$ Super-Weyl theory. It has the following form:

(i) A standard massless spin-two graviton multiplet with the following $\left(h, q_{R}\right)$ helicity $h$ components and $\mathrm{U}(1)_{R} q_{R}$ charges and with $n_{B}+n_{F}=4$ degrees of freedom:

$$
g_{\mathcal{N}=1}:(+2,0)+\left(+\frac{3}{2},+\frac{1}{2}\right)
$$

and its CPT conjugate

$$
\left(-\frac{3}{2},-\frac{1}{2}\right)+(-2,0)
$$

(ii) In the non-standard sector there is a massive spin-two supermultiplet, ${ }^{6}$ which is the socalled massive $\mathcal{N}=1$ super-Weyl multiplet $[54,55]$ with $n_{B}+n_{F}=16$ degrees of freedom:

$$
w_{\mathcal{N}=1}: \operatorname{Spin}(2)+\underline{2} \times \operatorname{Spin}(3 / 2)+\operatorname{Spin}(1)
$$

Note that the massive states in $\mathcal{N}=1$ supergravity built representations of the group $\operatorname{USp}(2)$.

Hence in summary, the massive super-(Weyl) $)^{2}$ gravity theory contains $n_{B}+n_{F}=20$ degrees of freedom.

\subsection{Massless theory}

The Weyl equation of motion eq. (2.10) now reads at linearized level [41]:

$$
D^{\alpha} \partial_{\dot{\beta}}^{\beta} \mathcal{W}_{\alpha \beta \gamma}=0 .
$$

This equation is equivalent to the linearized Weyl equation of motion of the bosonic Weyl tensor, as given in eq. (2.11). Then the spectrum of the massless $\mathcal{N}=1$ Super-Weyl theory has the following form:

\footnotetext{
${ }^{6}$ General massive multiplets in extended supersymmetry were discussed in [53].
} 
(i) A standard massless spin-two supergravity multiplet with $n_{B}+n_{F}=4$ degrees of freedom and the following $\left(h, q_{R}\right)$ helicity $h$ components and $\mathrm{U}(1)_{R} q_{R}$ charges:

$$
g_{\mathcal{N}=1}:(+2,0)+\left(+\frac{3}{2},+\frac{1}{2}\right)
$$

together with its CPT conjugate multiplet

$$
\left(-\frac{3}{2},-\frac{1}{2}\right)+(-2,0)
$$

Note that the $\mathrm{U}(1)_{R}$ charges and the helicities of the fermions are correlated, i.e. the states with positive helicity have also positive $\mathrm{U}(1)_{R}$ charge, and due to CPT the opposite is true for the states with negative helicities.

(ii) In the non-standard sector, we decompose the massive states into their massless helicity components. ${ }^{7}$ Furthermore we have to decompose the USp(2) representations into the $\mathrm{U}(1)_{R}$ charges of the massless states:

$$
\mathrm{USp}(2) \rightarrow \mathrm{U}(1)_{R}: \quad \underline{2}=\frac{1}{2} \oplus-\frac{1}{2},
$$

First, we get from the massive Weyl multiplet $w_{\mathcal{N}=1}$ a massless ghost-like spin-two supermultiplet:

$$
\text { spin }- \text { two : }(+2,0)+\left(+\frac{3}{2},+\frac{1}{2}\right),
$$

and its CPT conjugate as in (3.11). Second we get from $w_{\mathcal{N}=1}$ a massless, physical spin-3/2 supermultiplet:

$$
\operatorname{spin}-3 / 2:\left(+\frac{3}{2},-\frac{1}{2}\right)+(+1,0),
$$

together with its CPT conjugate multiplet

$$
(-1,0)+\left(-\frac{3}{2},+\frac{1}{2}\right)
$$

The spin-3/2 fields together build a so-called tripole ghost, which effectively acts as a physical spin-3/2 multiplet and a dipole ghost spin-2 multiplet [41].

And thirdly, $w_{\mathcal{N}=1}$ provides a physical, massless spin-one vector multiplet:

$$
\text { spin - one : }(+1,0)+\left(+\frac{1}{2},+\frac{1}{2}\right)
$$

and its CPT conjugate

$$
\left(-\frac{1}{2},-\frac{1}{2}\right)+(-1,0)
$$

\footnotetext{
${ }^{7}$ Note when decomposing massive into massless multiplets, the latter always come with their CPT conjugate multiplet because the massive ones are CPT invariant.
} 
Note that the massive Weyl multiplet $w_{\mathcal{N}=1}$ contains in addition a chiral spin- $1 / 2$ multiplet:

$$
\left(+\frac{1}{2},-\frac{1}{2}\right)+(0,0)
$$

and its CPT conjugate

$$
(0,0)+\left(-\frac{1}{2},+\frac{1}{2}\right)
$$

However this multiplet is unphysical since it can be gauge away by the superconformal transformations together with the local $\mathrm{U}(1)_{R}$ transformations. Specifically, one of the two scalars in this chiral multiplet is the Weyl mode, i.e. the helicity zero component of the massive spin-two field $w_{\mu \nu}$ in $w_{\mathcal{N}=1}$ and the other scalar is the helicity zero component of the massive vector inside $w_{\mathcal{N}=1}$, which is gauged away by the $\mathrm{U}(1)_{R}$ transformations.

Hence in summary, the massless super-(Weyl) $)^{2}$ gravity theory contains $n_{B}+n_{F}=16$ physical, propagating degrees of freedom [56]. Regarding the pure super-Weyl lagrangian in eq. (3.5), when the standard sugra term is omitted, the graviton \pm 1 states belong to the $\mathcal{N}=1$ vector multiplet (eqs. (3.16) and (3.17)), while the $A_{\mu}$ field is the gauge field of the $\mathrm{U}(1) \mathrm{R}$-symmetry and belongs to the spin-3/2 multiplet (eqs. (3.14) and (3.15)).

We note that, in accordance to [56], the gravitino action contains $(\mathcal{N}=1)$ eight helicity states, three (tripole ghost) $\left(+\frac{3}{2},+\frac{1}{2}\right),\left(+\frac{3}{2},+\frac{1}{2}\right),\left(+\frac{3}{2},-\frac{1}{2}\right)$ and a spin- $1 / 2$ state $\left(+\frac{1}{2},+\frac{1}{2}\right)(+\mathrm{CPT}$ conjugates). This phenomenon will be present for all $\mathcal{N}$, so the spectrum always contains the states $\left(+\frac{3}{2}, \mathcal{N}\right)\left(+\frac{3}{2}, \mathcal{N}\right),\left(+\frac{3}{2}, \overline{\mathcal{N}}\right),\left(+\frac{1}{2}, \mathcal{N}\right)+$ CPT conjugates, (the last comes from the gravitino multiplet for $\mathcal{N}=4)$. For $\mathcal{N}=4$ there are additional spin- $1 / 2$ states in the $4, \overline{4}$ and $\overline{20}$ of $\mathrm{SU}(4)$. The three states $\left(+\frac{1}{2}, \overline{4}\right),\left(+\frac{1}{2}, \overline{4}\right),\left(+\frac{1}{2}, 4\right)$ (the first two from the spin-2 multiplets and the last from the spin-3/2 multiplet) and their CPT conjugates, are described by a spin- $1 / 2$ cubic kinetic term, and finally the $\left(+\frac{1}{2}, \overline{20}\right)(+\mathrm{CPT}$ conjugate) is described by a standard (first derivative) kinetic term [58].

\subsection{Higher tensors}

Again, we like to consider some higher tensor in the massless $\mathcal{N}=1$ super-Weyl ${ }^{2}$ theory. We will restrict ourselves to construct the relevant superfields in the linearized approximation of the super-Weyl ${ }^{2}$ theory. First, the linearized super-Bel Robinson tensor has the following form:

$$
\mathcal{T}_{\alpha \beta \gamma, \dot{\alpha} \dot{\beta} \dot{\gamma}}=\mathcal{W}_{\alpha \beta \gamma} \overline{\mathcal{W}}_{\dot{\alpha} \dot{\beta} \dot{\gamma}}
$$

It is a tensor of spin $\left(\frac{3}{2}, \frac{3}{2}\right)$ and contains a bosonic spin-four field. The bosonic part of the super-Bel Robinson tensor is

$$
T_{\alpha \beta \gamma \delta, \dot{\alpha} \dot{\beta} \dot{\gamma} \dot{\delta}}=D_{\delta} \bar{D}_{\dot{\delta}} \mathcal{T}_{\alpha \beta \gamma, \dot{\alpha} \dot{\beta} \dot{\gamma}} \mid=W_{\alpha \beta \gamma \delta} \bar{W}_{\dot{\alpha} \dot{\beta} \dot{\gamma} \dot{\delta}} .
$$

Recalling that $W_{\alpha \beta \gamma \delta}$ and $W_{\dot{\alpha} \dot{\beta} \dot{\gamma} \dot{\delta}}$ correspond to the anti-self dual ${ }^{-} W^{\lambda}{ }_{\nu \mu \kappa}$ and the self dual ${ }^{+} W^{\lambda}{ }_{\nu \mu \kappa}$ parts of the Weyl tensor, we find the components of the Bel-Robinson tensor with Lorentz indices $T_{\mu \nu \rho \sigma}$ to be

$$
T_{\mu \nu \rho \sigma}={ }^{-} W_{\nu \mu \kappa}^{\lambda}{ }^{+} W_{\lambda \sigma \rho}{ }^{\kappa},
$$


where

$$
{ }^{ \pm} W_{\mu \nu \rho \sigma}=\frac{1}{2}\left(W_{\mu \nu \rho \sigma} \mp i{ }^{*} W_{\mu \nu \rho \sigma}\right)
$$

with

$$
{ }^{*} W_{\mu \nu \rho \sigma}=\frac{1}{2} \epsilon_{\mu \nu}{ }^{\kappa \lambda} W_{\kappa \lambda \rho \sigma} .
$$

Therefore, we may express (3.22) as

$$
T_{\mu \nu \rho \sigma}=\frac{1}{4}\left(W_{\nu \mu}^{\lambda}{ }_{\nu \mu}^{\kappa} W_{\lambda \sigma \rho \kappa}+{ }^{*} W_{\nu \mu}^{\lambda}{ }^{\kappa}{ }^{*} W_{\lambda \sigma \rho \kappa}\right)
$$

which coincides with the definition (2.12). Notice that due to eq. (3.1) the super-Bel Robinson superfield $\mathcal{T}_{\alpha \beta \gamma, \dot{\alpha} \dot{\beta} \dot{\gamma}}$ satisfies

$$
D^{\alpha} \mathcal{T}_{\alpha \beta \gamma, \dot{\alpha} \dot{\beta} \dot{\gamma}}=\bar{D}^{\dot{\alpha}} \mathcal{T}_{\alpha \beta \gamma, \dot{\alpha} \dot{\beta} \dot{\gamma}}=0,
$$

and therefore, it is conserved in Einstein supergravity.

$$
\partial^{\alpha \dot{\alpha}} \mathcal{T}_{\alpha \beta \gamma, \dot{\alpha} \dot{\beta} \dot{\gamma}}=0
$$

However in Weyl supergravity, this is not the case (at least from Bach-flat but not Ricciflat backgrounds) and therefore $T_{\alpha \beta \gamma, \dot{\alpha} \dot{\beta} \dot{\gamma}}$ is not the correct object we are looking for, as in addition it has dimension four.

As in the bosonic case, we are considering the super-Cotton tensor, which is given as

$$
\mathcal{C}_{\dot{\alpha} \beta \gamma}=\partial_{\dot{\alpha}}^{\alpha} \mathcal{W}_{\alpha \beta \gamma}
$$

Note that $\mathcal{C}_{\dot{\alpha} \beta \gamma}$ is symmetric in the last two indices $\beta$ and $\gamma$ and it has spin $\left(1, \frac{1}{2}\right)$. Using the super-Cotton tensor the linear super-Weyl equation of motion (3.9) can be rewritten as

$$
D^{\beta} \mathcal{C}_{\dot{\alpha} \beta \gamma}=0 \text {. }
$$

Finally we construct the super-version of the bosonic tensor $J_{\alpha \mu \nu}^{\lambda}$ in eq. (2.28). It has the following form

$$
\mathcal{J}_{\dot{\alpha} \beta \gamma, \alpha \dot{\beta} \dot{\gamma}}=\mathcal{C}_{\dot{\alpha} \beta \gamma} \overline{\mathcal{C}}_{\alpha \dot{\beta} \dot{\gamma}} .
$$

The tensor $J_{\dot{\alpha} \beta \gamma, \alpha \dot{\beta} \dot{\gamma}}$ has spin $\left(\frac{3}{2}+\frac{1}{2}, \frac{3}{2}+\frac{1}{2}\right)$. When writing this field in components, it contains a spin-four field, namely precisely $J^{\lambda \mu \nu \rho}$, which was introduced before. Indeed, we have that

$$
J_{\dot{\alpha} \beta \gamma \delta, \alpha \dot{\beta} \dot{\gamma} \dot{\delta}}=D_{\delta} \bar{D}_{\dot{\delta}} \mathcal{J}_{\dot{\alpha} \beta \gamma, \alpha \dot{\beta} \dot{\gamma}} \mid=C_{\dot{\alpha} \beta \gamma \delta} \bar{C}_{\alpha \dot{\beta} \dot{\gamma} \dot{\delta}}=\partial_{\dot{\alpha}}^{\alpha} W_{\alpha \beta \gamma \delta} \partial_{\alpha}^{\dot{\alpha}} W_{\dot{\alpha} \dot{\beta} \dot{\gamma} \dot{\delta}}
$$

Proceeding as above, we find that the corresponding Lorentz index tensor $J_{\nu \sigma \mu \rho}$ is given by

$$
J_{\nu \sigma \mu \rho}=\partial^{\lambda-} W_{\nu \mu \lambda}^{\kappa} \partial^{\tau+} W_{\kappa \sigma \rho \tau}=\frac{1}{4}\left(\partial^{\lambda} W^{\kappa}{ }_{\nu \mu \lambda} \partial^{\tau} W_{\kappa \sigma \rho \tau}+\partial^{\lambda *} W_{\nu \mu \lambda}^{\kappa} \partial^{\tau *} W_{\kappa \sigma \rho \tau}\right),
$$


which can be written in terms of the Cotton tensor as

$$
\begin{aligned}
J_{\nu \sigma \mu \rho} & =\frac{1}{4}\left(C^{\kappa}{ }_{\nu \mu} C_{\kappa \sigma \rho}+\frac{1}{4} \epsilon^{\kappa}{ }_{\nu}{ }^{\lambda \xi} \epsilon_{\kappa \sigma}{ }^{\chi \psi} C_{\lambda \xi \mu} C_{\chi \psi \rho}\right) \\
& =\frac{1}{4}\left(C^{\kappa}{ }_{\nu \mu} C_{\kappa \sigma \rho}+C^{\kappa}{ }_{\nu \rho} C_{\kappa \sigma \mu}-\frac{1}{2} g_{\nu \sigma} C^{\kappa \lambda} C_{\kappa \lambda \rho}\right),
\end{aligned}
$$

i.e., coincides with eq. (2.28)

However note that $J_{\dot{\alpha} \beta \gamma, \alpha \dot{\beta} \dot{\gamma}}$ is not irreducible in terms of Lorentz spins, but it also contains fields of lower spins:

$$
J_{\dot{\alpha} \beta \gamma, \alpha \dot{\beta} \dot{\gamma}}: \quad 3+3(2)+4(1)+2(0) .
$$

Using the super-Weyl equation of motion one can show that $J_{\dot{\alpha} \beta \gamma, \alpha \dot{\beta} \dot{\gamma}}$ is indeed covariantly conserved:

$$
D^{\beta} J_{\dot{\alpha} \beta \gamma, \alpha \dot{\beta} \dot{\gamma}}=D^{\dot{\beta}} J_{\dot{\alpha} \beta \gamma, \alpha \dot{\beta} \dot{\gamma}}=0 .
$$

Equation (3.35) can be rewritten as

$$
D^{\beta} J_{\dot{\alpha} \beta \gamma, \alpha \dot{\beta} \dot{\gamma}}=D_{\dot{\alpha}} S_{\dot{\beta} \gamma, \alpha \dot{\gamma}}=0
$$

$S_{\dot{\beta} \gamma, \alpha \dot{\gamma}}$ is a superfield with highest spin-2 component (and containing a bosonic spin-3 field), namely its spin content is as follows:

$$
S_{\dot{\beta} \gamma, \alpha \dot{\gamma}}: \quad\left(\frac{1}{2}, \frac{1}{2}\right) \otimes\left(\frac{1}{2}, \frac{1}{2}\right)=2+3(1)+2(0) .
$$

Therefore this conservation equation implies that these components in $J_{\dot{\alpha} \beta \gamma, \alpha \dot{\beta} \dot{\gamma}}$ get eliminated and the physical spin-components of $J_{\dot{\alpha} \beta \gamma, \alpha \dot{\beta} \dot{\gamma}}$ are the following:

$$
J_{\dot{\alpha} \beta \gamma, \alpha \dot{\beta} \dot{\gamma}}: \quad 3+2(2)+1 .
$$

\section{$4 \mathcal{N}=2$ super-Weyl theory}

\subsection{Massive theory}

The spectrum of the massive $\mathcal{N}=2$ Super-Weyl theory has the following form:

(i) A standard massless spin-two super graviton multiplet with $n_{B}+n_{F}=8$ degrees of freedom. It contains states with the following helicities and their associated $\mathrm{U}(2)$ quantum numbers:

$$
\left(2_{1}, \underline{1}_{0}\right)+\left(\frac{3}{2}, \underline{2}_{1}\right)+\left(1, \underline{1}_{2}\right)
$$

In addition there are the following CPT conjugate states

$$
\left(-1, \underline{1}_{-2}\right)+\left(-\frac{3}{2}, \underline{2}_{-1}\right)+\left(-2, \underline{1}_{0}\right) .
$$


(ii) In the non-standard sector we have a massive spin-two supermultiplet, which is the $\mathcal{N}=2$ massive super-Weyl multiplet [31] with $n_{B}+n_{F}=48$ degrees of freedom:

$$
w_{\mathcal{N}=2}: \operatorname{Spin}(2)+\underline{4} \times \operatorname{Spin}(3 / 2)+(\underline{5}+\underline{1}) \times \operatorname{Spin}(1)+\underline{4} \times \operatorname{Spin}(1 / 2)+\operatorname{Spin}(0) .
$$

Note that the massive states in $\mathcal{N}=2$ supergravity built representations of the group USp(4).

Hence in summary, the $\mathcal{N}=2$ massive super-(Weyl) ${ }^{2}$ gravity theory contains $n_{B}+n_{F}=56$ degrees of freedom.

\subsection{Massless theory}

Specifically the spectrum of the massless $\mathcal{N}=2$ Super-Weyl theory has the following form:

(i) A standard massless spin-two super graviton multiplet with $n_{B}+n_{F}=8$ degrees of freedom. Its helicity and $\mathrm{U}(2)$ quantum numbers are as in eqs. (4.1) and (4.2).

(ii) In the non-standard sector, we get first from the massive Weyl multiplet $w_{\mathcal{N}=2}$ a massless ghost-like spin-two supermultiplet with $n_{B}+n_{F}=8$. It contains states with helicity and $\mathrm{U}(2)$ quantum numbers, again as given in eqs. (4.1) and (4.2).

Second we get from $w_{\mathcal{N}=2}$ two massless spin-3/2 supermultiplets with in total $n_{B}+$ $n_{F}=16$ degrees of freedom:

$$
\underline{2}_{-1} \times\left[\left(\frac{3}{2}, \underline{1}_{0}\right)+\left(1, \underline{2}_{1}\right)+\left(\frac{1}{2}, \underline{1}_{2}\right)\right] .
$$

As before, there are the following additional CPT conjugate states

$$
\underline{2}_{1} \times\left[\left(-\frac{1}{2}, \underline{1}_{-2}\right)+\left(-1, \underline{2}_{-1}\right)+\left(-\frac{3}{2}, \underline{1}_{0}\right)\right] .
$$

Altogether they contain the four gauge bosons of the local $\mathrm{U}(2)_{R}$ gauge symmetry in the $\underline{1}_{0}+\underline{3}_{0}$ representations.

The massive Weyl multiplet $w_{\mathcal{N}=2}$ contains in addition two $\mathcal{N}=2$ vector multiplets. The first vector multiplet has the form:

$$
\left(1, \underline{1}_{0}\right)+\left(\frac{1}{2}, \underline{2}_{1}\right)+\left(0, \underline{1}_{2}\right) .
$$

Its CPT conjugate multiplet is given as

$$
\left(0, \underline{1}_{-2}\right)+\left(-\frac{1}{2}, \underline{2}_{-1}\right)+\left(-1, \underline{1}_{0}\right) .
$$

This vector multiplet is physical and contains propagating degrees of freedom. The neutral massless vector in this equation is the original \pm 1 helicity partner of the graviton. 
The second vector multiplet and its CPT conjugate possess different $\mathrm{U}(1)$ charges, namely

$$
\left(1, \underline{1}_{-2}\right)+\left(\frac{1}{2}, \underline{2}_{-1}\right)+\left(0, \underline{1}_{0}\right)
$$

and

$$
\left(0, \underline{1}_{0}\right)+\left(-\frac{1}{2}, \underline{2}_{1}\right)+\left(-1, \underline{1}_{2}\right)
$$

It contains a neutral scalar, namely the Weyl mode, which is gauged away by the conformal transformations. Hence this vector-multiplet gets removed, it is unphysical and does not propagate.

Finally the non-standard sector also contains one CPT self-conjugate, massless $\mathcal{N}=2$ hyper multiplet:

$$
\left(\frac{1}{2}, \underline{2}_{-1}\right)+\left(0, \underline{2}_{-1} \otimes 2_{1}\right)+\left(-\frac{1}{2}, \underline{2}_{1}\right)
$$

This hyper multiplet is unphysical, namely the four scalars in the $\underline{1}_{0}+\underline{3}_{0}$ representations are the helicity zero components of the massive vectors inside $w_{\mathcal{N}=2}$, which are gauged away by the local $\mathrm{U}(2)_{R}$ transformations.

Hence in summary, the massless $\mathcal{N}=2$ super-(Weyl) $)^{2}$ gravity theory contains $n_{B}+n_{F}=40$ physical, propagating degrees of freedom.

\section{$5 \mathcal{N}=3$ super-Weyl theory}

\subsection{Massive theory}

The spectrum of the massive $\mathcal{N}=3$ Super-Weyl theory has the following form:

(i) A standard massless spin-two super graviton multiplet $g_{\mathcal{N}=3}$ with $n_{B}+n_{F}=16$ degrees of freedom and the following helicity and $\mathrm{U}(3)$ quantum numbers:

$$
\left(2, \underline{1}_{0}\right)+\left(\frac{3}{2}, \underline{3}_{1}\right)+\left(1, \underline{3}_{2}\right)+\left(\frac{1}{2}, \underline{1}_{3}\right) .
$$

In addition one obtains the CPT conjugate states:

$$
\left(-\frac{1}{2}, \underline{1}_{-3}\right)+\left(-1, \underline{3}_{-2}\right)+\left(-\frac{3}{2}, \underline{\overline{3}}_{-1}\right)+\left(-2, \underline{1}_{0}\right)
$$

(ii) In the non-standard sector we have a massive spin-two supermultiplet, which is the $\mathcal{N}=3$ massive super-Weyl multiplet with $n_{B}+n_{F}=128$ degrees of freedom [57]:

$$
\begin{aligned}
w_{\mathcal{N}=3}: & \operatorname{Spin}(2)+\underline{6} \times \operatorname{Spin}(3 / 2)+(\underline{14}+\underline{1}) \times \operatorname{Spin}(1)+\left(\underline{14^{\prime}}+\underline{6}\right) \times \operatorname{Spin}(1 / 2) \\
& +\underline{14} \times \operatorname{Spin}(0) .
\end{aligned}
$$

Note that the massive states in $\mathcal{N}=3$ supergravity built representations of the group $\operatorname{USp}(6)$.

Hence in summary, the $\mathcal{N}=3$ massive super-(Weyl) ${ }^{2}$ gravity theory contains $n_{B}+n_{F}=144$ degrees of freedom. 


\subsection{Massless theory}

The spectrum of the massless $\mathcal{N}=3$ Super-Weyl theory has the following form:

(i) A standard massless spin-two super graviton multiplet with $n_{B}+n_{F}=16$ degrees of freedom, as given in eqs. (5.1) and (5.2).

(ii) In the non-standard sector, in order to obtain the massless states, we have to decompose the massive representations into massless representations. This is done via the branching rules of the massive USp(6) R-symmetry group into the R-symmetry group $\mathrm{U}(3)$ of the massless states. The specific decomposition of $\mathrm{USp}(6) \rightarrow \mathrm{SU}(3) \times \mathrm{U}(1)$ for the relevant representations is as follows:

$$
\begin{aligned}
\underline{6} & =\underline{3}_{1} \oplus \underline{\overline{3}}_{-1}, \\
\underline{14} & =\underline{3}_{-2} \oplus \underline{\overline{3}}_{2} \oplus \underline{8}_{0}, \\
\underline{14^{\prime}} & =\underline{1}_{3} \oplus \underline{1}_{-3} \oplus \underline{6}_{-1} \oplus \underline{\overline{6}}_{1} .
\end{aligned}
$$

Then we get first from the massive Weyl multiplet $w_{\mathcal{N}=3}$ a spin-two supermultiplet, again with the states as given eqs. (5.1) and (5.2). They constitute a massless ghostlike spin-two supermultiplet with $n_{B}+n_{F}=16$.

Second we get from $w_{\mathcal{N}=3}$ a helicity $+3 / 2$ supermultiplet with the $\mathrm{U}(3)$ charge assignements

$$
\underline{\overline{3}}_{-1} \otimes\left[\left(\frac{3}{2}, \underline{1}_{0}\right)+\left(1, \underline{3}_{1}\right)+\left(\frac{1}{2}, \underline{3}_{2}\right)+\left(0, \underline{1}_{3}\right)\right],
$$

plus the CPT conjugate multiplet of the form

$$
\underline{3}_{1} \otimes\left[\left(0, \underline{1}_{-3}\right)+\left(-\frac{1}{2}, \underline{\overline{3}}_{-2}\right)+\left(-1, \underline{\overline{3}}_{-1}\right)+\left(-\frac{3}{2}, \underline{1}_{0}\right)\right] .
$$

These two multiplets together build three massless, physical spin-3/2 supermultiplets with in total $n_{B}+n_{F}=48$. They contain the nine gauge bosons, which transform as $\underline{1}_{0}+\underline{8}_{0}$, of the local $\mathrm{U}(3)_{R}$ gauge symmetry.

Finally the massive Weyl multiplet $w_{\mathcal{N}=3}$ contains in addition the following helicity states:

$$
\left(\underline{1}_{0}+\underline{3}_{-2}\right) \otimes\left[\left(1, \underline{1}_{0}\right)+\left(\frac{1}{2}, \underline{3}_{1}\right)+\left(0, \underline{\overline{3}}_{2}\right)+\left(-\frac{1}{2}, \underline{1}_{3}\right)\right] .
$$

There are also the CPT conjugate states of the form:

$$
\left(\underline{1}_{0}+\underline{3}_{2}\right) \otimes\left[\left(\frac{1}{2}, \underline{1}_{-3}\right)+\left(0, \underline{3}_{-2}\right)+\left(-\frac{1}{2}, \underline{\overline{3}}_{-1}\right)+\left(-1, \underline{1}_{0}\right)\right] .
$$

Altogether the states in eqs. (5.7) and (5.8) built four full, massless $\mathcal{N}=3$ vectormultiplets. The 24 scalars in these four vector multiplets transform under $\mathrm{U}(3)$ as $\underline{1}_{0}+\underline{1}_{0}+\underline{3}_{-2}+\underline{\overline{3}}_{2}+\underline{8}_{0}+\underline{8}_{0}$. One singlet scalar is gauged away by the conformal transformations. Furthermore nine scalars in $\underline{1}_{0}+\underline{8}_{0}$ representations are the helicity 
zero component of the massive vectors inside $w_{\mathcal{N}=3}$, which are gauged away by the local $\mathrm{U}(3)_{R}$ transformations. It follows that three of the four vector multiplets get removed. They are unphysical and correspond to superconformal and U(3) gauge degrees of freedom. Therefore one gets just one physical, propagating, massless $\mathcal{N}=3$ vector multiplet with the following fields:

$$
\left(1, \underline{1}_{0}\right)+\left(\frac{1}{2}, \underline{3}_{1}\right)+\left(0, \underline{3}_{2}\right)+\left(-\frac{1}{2}, \underline{1}_{3}\right)
$$

together with its CPT conjugate state.

Hence in summary, the massless $\mathcal{N}=3$ super-(Weyl) $)^{2}$ gravity theory contains $n_{B}+n_{F}=96$ physical, propagating degrees of freedom.

\section{$6 \mathcal{N}=4$ super-Weyl theory}

\subsection{Massive theory}

The spectrum of the massive $\mathcal{N}=4$ Super-Weyl theory has the following form:

(i) A standard massless spin-two super graviton multiplet $g_{\mathcal{N}=4}$ with $n_{B}+n_{F}=32$ degrees of freedom and with the following helicities and SU(4) representations:

$$
(+2, \underline{1})+\left(+\frac{3}{2}, \underline{4}\right)+(1, \underline{6})+\left(+\frac{1}{2}, \overline{4}\right)+(0, \underline{1}),
$$

together with its CPT conjugate

$$
(0, \underline{1})+\left(-\frac{1}{2}, \underline{4}\right)+(-1, \underline{6})+\left(-\frac{3}{2}, \underline{\overline{4}}\right)+(-2, \underline{1})
$$

(ii) In the non-standard sector we have the spin-two massive Weyl multiplet of $\mathcal{N}=4$, which is irreducible with $n_{B}+n_{F}=2^{8}=256$ with states in $\operatorname{USp}(8)$ representations [58]:

$$
w_{\mathcal{N}=4}: \operatorname{Spin}(2)+\underline{8} \times \operatorname{Spin}(3 / 2)+\underline{27} \times \operatorname{Spin}(1)+\underline{48} \times \operatorname{Spin}(1 / 2)+\underline{42} \times \operatorname{Spin}(0) .
$$

Hence in summary, the $\mathcal{N}=4$ massive super-(Weyl) $)^{2}$ gravity theory contains $n_{B}+n_{F}=288$ degrees of freedom.

\subsection{Massless theory}

Now we need the branching rules of the massive USp(8) R-symmetry group into the Rsymmetry group SU(4) of the massless states. The specific decomposition of USp(8) $\rightarrow$ $\mathrm{SU}(4)$ for the relevant representations is as follows:

$$
\begin{aligned}
\underline{8} & =\underline{4} \oplus \underline{\overline{4}}, \\
\underline{27} & =\underline{6} \oplus \underline{\overline{6}} \oplus \underline{15}, \\
\underline{42} & =\underline{1} \oplus \underline{\overline{1}} \oplus \underline{10}+\underline{\overline{10}} \oplus \underline{20^{\prime}}, \\
\underline{48} & =\underline{20} \oplus \underline{\underline{20}} \oplus \underline{4} \oplus \underline{\overline{4}}
\end{aligned}
$$

Then the spectrum of the massless $\mathcal{N}=4$ Super-Weyl theory has the following form: 
(i) A standard massless spin-two supergravity multiplet with $n_{B}+n_{F}=32$ degrees of freedom as given in eqs. (6.1) and (6.2).

(ii) In the non-standard sector, we get first from the massive Weyl multiplet $w_{\mathcal{N}=4}$ a massless ghost-like spin-two supermultiplet with $n_{B}+n_{F}=32$ and with the helicites and $\mathrm{SU}(4)$ quantum numbers, again as given eqs. (6.1) and (6.2).

Second we get from $w_{\mathcal{N}=4}$ four massless spin-3/2 supermultiplets (in total $n_{B}+n_{F}=$ 128) with the following helicities and $\mathrm{SU}(4)$ representations, namely ${ }^{8}$

$$
\underline{\overline{4}} \times\left[\left(\frac{3}{2}, \underline{1}\right)+(1, \underline{4})+\left(\frac{1}{2}, \underline{6}\right)+(0, \underline{\overline{4}})+\left(-\frac{1}{2}, \underline{1}\right)\right],
$$

together with the CPT conjugate states

$$
\underline{4} \times\left[\left(\frac{1}{2}, \underline{1}\right)+(0, \underline{4})+\left(-\frac{1}{2}, \underline{6}\right)+(-1, \underline{\overline{4}})+\left(-\frac{3}{2}, \underline{1}\right)\right] .
$$

They contain the 15 gauge bosons of the local $\mathrm{SU}(4)_{R}$ gauge symmetry.

In addition, the massive Weyl multiplet $w_{\mathcal{N}=4}$ contains $\operatorname{six} \mathcal{N}=4$ vector multiplets of the form:

$$
6(\operatorname{spin}-\text { one }): \underline{6} \times\left[(+1, \underline{1})+\left(+\frac{1}{2}, \underline{4}\right)+(0, \underline{6})+\left(-\frac{1}{2}, \overline{4}\right)+(-1, \underline{1}) .\right]
$$

However these multiplets are unphysical since they can be gauged away by the superconformal transformations together with the local SU $(4)_{R}$ transformations. Specifically, one of the 36 scalars in these vector multiplets is a Weyl mode. Other 15 scalars are the helicity zero component of the massive vectors inside $w_{\mathcal{N}=4}$, which are gauged away by the local $\mathrm{SU}(4)_{R}$ transformations. Hence all six vector-multiplets are unphysical, do not propagate and get removed from the spectrum.

We should note that the dipole ghost graviton and the tripole ghost spin-3/2 sector are accompanied by a dipole ghost complex scalar since the action is a higher-derivative action. Indeed, the equations of motion are fourth-order for the spin-2 and third order for the spin-3/2 states. This fact is also discussed in [38, 39] at the Lagrangian level. This is not the case for the $\mathrm{SU}(4)$ gauge bosons which have standard Yang Mills action. The sugra higher derivative action also contains a singlet vector mode which, together with the gauge bosons, is part of the higher derivative gravitino action (which as pointed out above obeys third order equations of motion). In other words, the cubic gravitino action simultaneously describes the gravitino, the partner of the graviton, as well as the gravitini of the gravitino multiplet.

Hence in summary, the massless $\mathcal{N}=4$ super-(Weyl) ${ }^{2}$ gravity theory contains $n_{B}+$ $n_{F}=192$ physical, propagating degrees of freedom. The same spectrum was also obtained in [59] using the string twistor formalism for the construction of $\mathcal{N}=4$ super-(Weyl) ${ }^{2}$ gravity.

\footnotetext{
${ }^{8}$ The group theory of the massive AdS graviton Higgsing was discussed before in [60].
} 


\section{Conclusions and outlook}

In this paper we obtained the spectrum of all existing $\mathcal{N}$-extended $\mathrm{Weyl}^{2}$ supergravities $(\mathcal{N}=1,2,3,4)$ in four space-time dimensions. We are summarizing the physical spectrum, ie. the number of super-multiplets of the massless theories after subtracting the gauge multiplets, in the following table, where we indicate in the first column the top helicities of each multiplet together with its CPT conjugate multiplet:

\begin{tabular}{|c||c|c|c|c|}
\hline & $\mathcal{N}=4$ & $\mathcal{N}=3$ & $\mathcal{N}=2$ & $\mathcal{N}=1$ \\
\hline \hline$h_{\max }=2$ & 2 & & & \\
$h_{\max }^{\mathrm{CPT}}=0$ & 2 & & & \\
\hline$h_{\max }=3 / 2$ & 4 & & & \\
$h_{\max }^{\mathrm{CPT}}=1 / 2$ & 4 & & & \\
\hline \hline$h_{\max }=2$ & & 2 & & \\
$h_{\max }^{\mathrm{CPT}}=-1 / 2$ & & 2 & & \\
\hline$h_{\max }=3 / 2$ & & 3 & & \\
$h_{\max }^{\mathrm{CPT}}=0$ & & 3 & & \\
\hline$h_{\max }=1$ & & 1 & & \\
$h_{\max }^{\mathrm{CPT}}=1 / 2$ & & 1 & & \\
\hline \hline$h_{\max }=2$ & & & 2 & \\
$h_{\max }^{\mathrm{CPT}}=-1$ & & & 2 & \\
\hline$h_{\max }=3 / 2$ & & & 2 & \\
$h_{\max }^{\mathrm{CPT}}=-1 / 2$ & & & 2 & \\
\hline$h_{\max }=1$ & & & 1 & \\
$h_{\max }^{\mathrm{CPT}}=0$ & & & 1 & \\
\hline \hline$h_{\max }=2$ & & & & 2 \\
$h_{\max }^{\mathrm{CPT}}=-3 / 2$ & & & & 2 \\
\hline$h_{\max }=3 / 2$ & & & & 1 \\
$h_{\max }^{\mathrm{CPT}}=-1$ & & & & 1 \\
\hline$h_{\max }=1$ & & & & 1 \\
$h_{\max }^{\mathrm{CPT}}=-1 / 2$ & & & & 1 \\
\hline
\end{tabular}

Note that in the supersymmetric Weyl ${ }^{2}$ theories one can have bosonic terms with four derivatives and also some with only two derivatives. This happens when the two-derivative terms would be auxiliary fields in Einstein supergravity. In particular this is the case in $\mathrm{U}(\mathcal{N})$ Weyl $^{2}$ supergravity, where the Langrangian for the $\mathrm{U}(\mathcal{N})$ vector bosons is given by the canonical $F^{\mu \nu} F_{\mu \nu}$ term. ${ }^{9}$ In addition we also discussed some operators of higher dimension, corresponding to the Bell-Robinson tensor, which is basically the square of the Weyl tensor, or the square of the Cotton tensor.

${ }^{9}$ An analogous effect can be seen from the Wess-Zumino Langrangian

$$
\mathcal{L}=-\frac{1}{2} \partial^{\mu} z \square \partial_{\mu} \bar{z}-\frac{i}{2} \bar{\psi} \not \not^{3} \psi+\frac{1}{2} \bar{F} \square F,
$$

which contains three Wess-Zumino multiplets: the first term is a dipole spin 0 , the second is a tripole ghost spin $1 / 2$, whereas the last term describes two standard scalars. 
Another issue we would like to point out here is that the (super)-conformal symmetry of the (super)-Weyl ${ }^{2}$ theory is a classical symmetry. It is true that these theories are power-counting renormalizable but the one-loop beta-functions turns out to be nonvanishing [61] and therefore they suffer from a conformal anomaly. The latter leads to serious problems in Weyl gravity as the conformal symmetry is gauged and therefore leads to inconsistencies [62-64]. Let us also note that one may arrive at the same conclusion by the calculation of the chiral gauge anomalies of the SU(4) R-symmetry [65] and by recalling that all anomalies are arranged in the same multiplet of the $\mathcal{N}=4$ superconformal symmetry.

However, surprisingly, the $\mathcal{N}=4$ super-Weyl ${ }^{2}$ gravity can be made UV-finite [63, 66], and thus anomaly-free [65] as well. For $\mathcal{N}=4$ Poincaré supergravity it has been conjectured in [67] a hidden superconformal symmetry. This can be achieved by appropriate coupling the Weyl ${ }^{2}$ supergravity to four vector supermultiplets. Although this result has been shown to hold at one-loop level, it is expected to hold to all loops since the contributions to the $\beta$ function and the conformal anomaly of SYM is only an one-loop effect for $\mathcal{N}>1$ conformal supergravities [66]. For the $\mathcal{N}=4$ case in particular, the above statement is strengthen by the fact that the conformal anomaly is connected by supersymmetry to the SU(4) chiral anomaly which arises at one-loop also.

Let us notice that super-Weyl ${ }^{2}$ gravity is not the only superconformal theory. As it is very well known, the $\mathcal{N}=4 \mathrm{SYM}$ theory is also invariant under the rigid superconformal supergroup $\mathrm{SU}(2,2 \mid 4)$. Therefore, it can naturally source the $\mathcal{N}=4$ super-Weyl ${ }^{2}$ gravity. In addition, the latter may affect the standard AdS/CFT holography. Indeed, allowing a boundary $\mathrm{Weyl}^{2}$ operator still preserves the superconformal symmetry and it may correspond to a deformation of the holographic bulk theory.

Moreover, it could be, as pointed out in [68] that the pure $\mathcal{N}$-extended (Weyl) ${ }^{2}$ supergravity theory in four dimensions is the holographically dual boundary theory of an $A d S_{5}$ bulk theory, which is a higher spin theory with a spin-four multiplet of the $2 \mathcal{N}$ extended supersymmetry algebra in five dimensions. These kind of theories, denoted by $\mathrm{W}$-supergravities, were recently constructed [68] in flat four-dimensional space-time using a double copy and S-fold construction. Within such a duality, one would expect that a conformal operator respectively higher tensor $T_{\mu \nu \rho \sigma}$ on the boundary, which is quadratic in the curvature tensors and their derivatives, and which is also divergence-free on-shell, is coupled to a spin-four field in the bulk. It would be interesting to find the exact 5D holographic dual, if any, of the 4D Weyl supergravity.

Alternatively, one may consider super-Weyl ${ }^{2}$ gravity as a bulk theory. In that case, $A d S_{4}$ is still a vacuum solution of the theory and there should exists a holographic 3D theory in the boundary, which it might not be unitary. Indeed, the massless graviton will still be coupled to a conserved spin-2 operator (energy-momentum tensor) at the boundary, the massless vector will be coupled to a conserved spin-1 operator, whereas it is expected that the massive spin-2 ghost to couple to a non-unitary spin-2 operator. Furthermore, it would be very interesting to see if there is a corresponding string construction of holographic duality, possibly using the twistor string approach of [59] for the construction of the superconformal Weyl ${ }^{2}$ theory. 


\section{Acknowledgments}

We gratefully acknowledge correspondence with N. Boulanger and F. Farakos. The work of S.F. is supported in part by CERN TH Dept and INFN-CSN4-GSS. The work of D.L. is supported by the ERC Advanced Grant "Strings and Gravity" (Grant No. 320045) and the Excellence Cluster Universe. He also is grateful to the CERN theory department for its hospitality, when part of this work was performed. A.K. is supported by the GSRT under the EDEIL/67108600.

Open Access. This article is distributed under the terms of the Creative Commons Attribution License (CC-BY 4.0), which permits any use, distribution and reproduction in any medium, provided the original author(s) and source are credited.

\section{References}

[1] K.S. Stelle, Renormalization of Higher Derivative Quantum Gravity, Phys. Rev. D 16 (1977) 953 [INSPIRE].

[2] K.S. Stelle, Classical Gravity with Higher Derivatives, Gen. Rel. Grav. 9 (1978) 353 [INSPIRE].

[3] D.G. Boulware, G.T. Horowitz and A. Strominger, Zero Energy Theorem for Scale Invariant Gravity, Phys. Rev. Lett. 50 (1983) 1726 [inSPIRE].

[4] F. David and A. Strominger, On the Calculability of Newton's Constant and the Renormalizability of Scale Invariant Quantum Gravity, Phys. Lett. 143B (1984) 125 [INSPIRE].

[5] G.T. Horowitz, Quantum Cosmology With a Positive Definite Action, Phys. Rev. D 31 (1985) 1169 [inSPIRE].

[6] S. Deser and B. Tekin, Shortcuts to high symmetry solutions in gravitational theories, Class. Quant. Grav. 20 (2003) 4877 [gr-qc/0306114] [INSPIRE].

[7] S. Deser and B. Tekin, New energy definition for higher curvature gravities, Phys. Rev. D 75 (2007) 084032 [gr-qc/0701140] [inSPIRE].

[8] G. 't Hooft, A class of elementary particle models without any adjustable real parameters, Found. Phys. 41 (2011) 1829 [arXiv:1104.4543] [INSPIRE].

[9] J. Maldacena, Einstein Gravity from Conformal Gravity, arXiv:1105.5632 [INSPIRE].

[10] H. Lü, C.N. Pope, E. Sezgin and L. Wulff, Critical and Non-Critical Einstein-Weyl Supergravity, JHEP 10 (2011) 131 [arXiv:1107.2480] [INSPIRE].

[11] L. Álvarez-Gaumé, A. Kehagias, C. Kounnas, D. Lüst and A. Riotto, Aspects of Quadratic Gravity, Fortsch. Phys. 64 (2016) 176 [arXiv: 1505.07657] [INSPIRE].

[12] S. Cecotti, Higher derivative supergravity is equivalent to standard supergravity coupled to matter. 1., Phys. Lett. B 190 (1987) 86 [INSPIRE].

[13] S. Cecotti, S. Ferrara, M. Porrati and S. Sabharwal, New minimal higher derivative supergravity coupled to matter, Nucl. Phys. B 306 (1988) 160 [InSPIRE].

[14] R. Kallosh and A. Linde, Superconformal generalizations of the Starobinsky model, JCAP 06 (2013) 028 [arXiv: 1306.3214] [INSPIRE]. 
[15] J. Ellis, D.V. Nanopoulos and K.A. Olive, No-Scale Supergravity Realization of the Starobinsky Model of Inflation, Phys. Rev. Lett. 111 (2013) 111301 [Erratum ibid. 111 (2013) 129902] [arXiv: 1305.1247] [INSPIRE].

[16] J. Ellis, D.V. Nanopoulos and K.A. Olive, Starobinsky-like Inflationary Models as Avatars of No-Scale Supergravity, JCAP 10 (2013) 009 [arXiv:1307.3537] [INSPIRE].

[17] F. Farakos, A. Kehagias and A. Riotto, On the Starobinsky Model of Inflation from Supergravity, Nucl. Phys. B 876 (2013) 187 [arXiv: 1307.1137] [InSPIRE].

[18] S. Ferrara, R. Kallosh, A. Linde and M. Porrati, Minimal Supergravity Models of Inflation, Phys. Rev. D 88 (2013) 085038 [arXiv:1307.7696] [INSPIRE].

[19] R. Kallosh, A. Linde and D. Roest, Superconformal Inflationary $\alpha$-Attractors, JHEP 11 (2013) 198 [arXiv:1311.0472] [INSPIRE].

[20] J. Alexandre, N. Houston and N.E. Mavromatos, Starobinsky-type Inflation in Dynamical Supergravity Breaking Scenarios, Phys. Rev. D 89 (2014) 027703 [arXiv:1312.5197] [INSPIRE].

[21] S. Ferrara and M. Porrati, Minimal $R+R^{2}$ Supergravity Models of Inflation Coupled to Matter, Phys. Lett. B 737 (2014) 135 [arXiv:1407.6164] [INSPIRE].

[22] A. Ceresole, G. Dall'Agata, S. Ferrara, M. Trigiante and A. Van Proeyen, A search for an $\mathcal{N}=2$ inflaton potential, Fortsch. Phys. 62 (2014) 584 [arXiv:1404.1745] [InSPIRE].

[23] C. Kounnas, D. Lüst and N. Toumbas, $R^{2}$ inflation from scale invariant supergravity and anomaly free superstrings with fluxes, Fortsch. Phys. 63 (2015) 12 [arXiv:1409.7076] [INSPIRE].

[24] I. Dalianis, F. Farakos, A. Kehagias, A. Riotto and R. von Unge, Supersymmetry Breaking and Inflation from Higher Curvature Supergravity, JHEP 01 (2015) 043 [arXiv: 1409.8299] [INSPIRE].

[25] T. Terada, Y. Watanabe, Y. Yamada and J. Yokoyama, Reheating processes after Starobinsky inflation in old-minimal supergravity, JHEP 02 (2015) 105 [arXiv:1411.6746] [INSPIRE].

[26] S. Ferrara and A. Kehagias, Higher Curvature Supergravity, Supersymmetry Breaking and Inflation, Subnucl. Ser. 52 (2017) 119 [arXiv:1407.5187] [INSPIRE].

[27] S. Ferrara, A. Kehagias and A. Sagnotti, Cosmology and Supergravity, Int. J. Mod. Phys. A 31 (2016) 1630044 [arXiv:1605.04791] [INSPIRE].

[28] A.A. Starobinsky, A New Type of Isotropic Cosmological Models Without Singularity, Phys. Lett. B 91 (1980) 99 [INSPIRE].

[29] V.F. Mukhanov and G.V. Chibisov, Quantum Fluctuations and a Nonsingular Universe (in Russian), JETP Lett. 33 (1981) 532 [inSPIRE].

[30] S. Ferrara, M.T. Grisaru and P. van Nieuwenhuizen, Poincaré and Conformal Supergravity Models With Closed Algebras, Nucl. Phys. B 138 (1978) 430 [INSPIRE].

[31] B. de Wit, J.W. van Holten and A. Van Proeyen, Transformation Rules of $N=2$ Supergravity Multiplets, Nucl. Phys. B 167 (1980) 186 [INSPIRE].

[32] B. de Wit and S. Ferrara, On Higher Order Invariants in Extended Supergravity, Phys. Lett. B 81 (1979) 317. 
[33] S. Ferrara, M. Kaku, P.K. Townsend and P. van Nieuwenhuizen, Gauging the Graded Conformal Group with Unitary Internal Symmetries, Nucl. Phys. B 129 (1977) 125 [INSPIRE].

[34] M. Kaku, P.K. Townsend and P. van Nieuwenhuizen, Superconformal Unified Field Theory, Phys. Rev. Lett. 39 (1977) 1109 [INSPIRE].

[35] M. Kaku, P.K. Townsend and P. van Nieuwenhuizen, Properties of Conformal Supergravity, Phys. Rev. D 17 (1978) 3179 [INSPIRE].

[36] D.Z. Freedman and A. Van Proeyen, Supergravity, Cambridge University Press, Cambridge U.K. (2012).

[37] J.M. Cline, S. Jeon and G.D. Moore, The Phantom menaced: Constraints on low-energy effective ghosts, Phys. Rev. D 70 (2004) 043543 [hep-ph/0311312] [INSPIRE].

[38] H. Johansson and J. Nohle, Conformal Gravity from Gauge Theory, arXiv:1707.02965 [INSPIRE].

[39] H. Johansson, G. Mogull and F. Teng, Unraveling conformal gravity amplitudes, arXiv: 1806.05124 [INSPIRE].

[40] A. Salvio, Quadratic Gravity, Front. in Phys. 6 (2018) 77 [arXiv:1804.09944] [InSPIRE].

[41] S. Ferrara and B. Zumino, Structure of Conformal Supergravity, Nucl. Phys. B 134 (1978) 301 [InSPIRE].

[42] R.J. Riegert, The particle content of linearized conformal gravity, Phys. Lett. A 105 (1984) 110 [INSPIRE].

[43] A. Balfagon and X. Jaen, Review of some classical gravitational superenergy tensors using computational techniques, Class. Quant. Grav. 17 (2000) 2491 [gr-qc/9912060] [INSPIRE].

[44] L. Bel, Sur la radiation gravitationelle, CR Acad. Sci. Paris 247 (1958) 1094.

[45] L. Bel, Introduction d'un tenseur du quatrieme order, CR Acad. Sci. Paris 248 (1959) 1297.

[46] I. Robinson, unpublished Kings College Lectures, London U.K. (1958).

[47] I. Robinson, On the Bel-Robinson tensor, Class. Quant. Grav. 14 (1997) 4331.

[48] S. Ferrara, R. Gatto and A.F. Grillo, Positivity Restrictions on Anomalous Dimensions, Phys. Rev. D 9 (1974) 3564 [INSPIRE].

[49] G. Mack, All unitary ray representations of the conformal group $\mathrm{SU}(2,2)$ with positive energy, Commun. Math. Phys. 55 (1977) 1 [INSPIRE].

[50] C. Bachas and I. Lavdas, Massive Anti-de Sitter Gravity from String Theory, arXiv: 1807.00591 [INSPIRE].

[51] N. Boulanger, A Weyl-covariant tensor calculus, J. Math. Phys. 46 (2005) 053508 [hep-th/0412314] [INSPIRE].

[52] F. Farakos, S. Ferrara, A. Kehagias and D. Lüst, Non-linear Realizations and Higher Curvature Supergravity, Fortsch. Phys. 65 (2017) 1700073 [arXiv:1707.06991] [INSPIRE].

[53] S. Ferrara, C.A. Savoy and B. Zumino, General Massive Multiplets in Extended Supersymmetry, Phys. Lett. 100B (1981) 393 [INSPIRE].

[54] S. Ferrara and B. Zumino, Transformation Properties of the Supercurrent, Nucl. Phys. B 87 (1975) 207 [InSPIRE]. 
[55] S. Ferrara and B. Zumino, Structure of Conformal Supergravity, Nucl. Phys. B 134 (1978) 301 [inSPIRE].

[56] S.C. Lee and P. van Nieuwenhuizen, Counting of States in Higher Derivative Field Theories, Phys. Rev. D 26 (1982) 934 [inSPIRE].

[57] J. van Muiden and A. Van Proeyen, The $\mathcal{N}=3$ Weyl Multiplet in Four Dimensions, arXiv:1702.06442 [INSPIRE].

[58] E. Bergshoeff, M. de Roo and B. de Wit, Extended Conformal Supergravity, Nucl. Phys. B 182 (1981) 173 [INSPIRE].

[59] N. Berkovits and E. Witten, Conformal supergravity in twistor-string theory, JHEP 08 (2004) 009 [hep-th/0406051] [INSPIRE].

[60] C. Bachas, M. Bianchi and A. Hanany, $\mathcal{N}=2$ moduli of AdS $S_{4}$ vacua: a fine-print study, JHEP 08 (2018) 100 [arXiv:1711.06722] [INSPIRE].

[61] E.S. Fradkin and A.A. Tseytlin, One Loop $\beta$-function in Conformal Supergravities, Nucl. Phys. B 203 (1982) 157 [inSPIRE].

[62] D.M. Capper and M.J. Duff, Conformal Anomalies and the Renormalizability Problem in Quantum Gravity, Phys. Lett. A 53 (1975) 361 [INSPIRE].

[63] E.S. Fradkin and A.A. Tseytlin, Conformal Anomaly in Weyl Theory and Anomaly Free Superconformal Theories, Phys. Lett. 134B (1984) 187 [INSPIRE].

[64] A.A. Tseytlin, On divergences in non-minimal $N=4$ conformal supergravity, J. Phys. A 50 (2017) 48LT01 [arXiv:1708.08727] [inSPIRE].

[65] H. Romer and P. van Nieuwenhuizen, Axial Anomalies in $N=4$ Conformal Supergravity, Phys. Lett. 162B (1985) 290 [INSPIRE].

[66] E.S. Fradkin and A.A. Tseytlin, Conformal supergravity, Phys. Rept. 119 (1985) 233 [INSPIRE].

[67] S. Ferrara, R. Kallosh and A. Van Proeyen, Conjecture on hidden superconformal symmetry of $N=4$ Supergravity, Phys. Rev. D 87 (2013) 025004 [arXiv:1209.0418] [INSPIRE].

[68] S. Ferrara and D. Lüst, Spin-four $\mathcal{N}=7 \mathrm{~W}$-supergravity: S-fold and double copy construction, JHEP 07 (2018) 114 [arXiv: 1805.10022] [INSPIRE]. 